\title{
Safety pin in human heart: An unusual distraction
}

\author{
Jacek Kurzawski ${ }^{1}$, Agnieszka Janion-Sadowska ${ }^{1}$, Marcin Sadowski ${ }^{1,2}$ \\ ${ }^{1}$ Swietokrzyskie Cardiology Centre, Kielce, Poland \\ ${ }^{2}$ Jan Kochanowski University, Kielce, Poland
}

A 60-year-old male serving a long-term prison sentence, heavy drinker and smoker was admitted after an episode of chest pain preceding syncope. Initial electrocardiogram was inconclusive. Physical examination and laboratory findings were within normal limits. On admission chest X-ray revealed a linear metallic foreign body in the left ventricular plane (Fig. 1A). Patient reported that he self-harmed in order to get out of prison about 30 years ago. He used a safety pin, unhinged into a straight metal piece to stab into the right lung. For this purpose he held the point of the pin at a right angle at the level of the intercostal space and hit the other end with the bottom of a metal

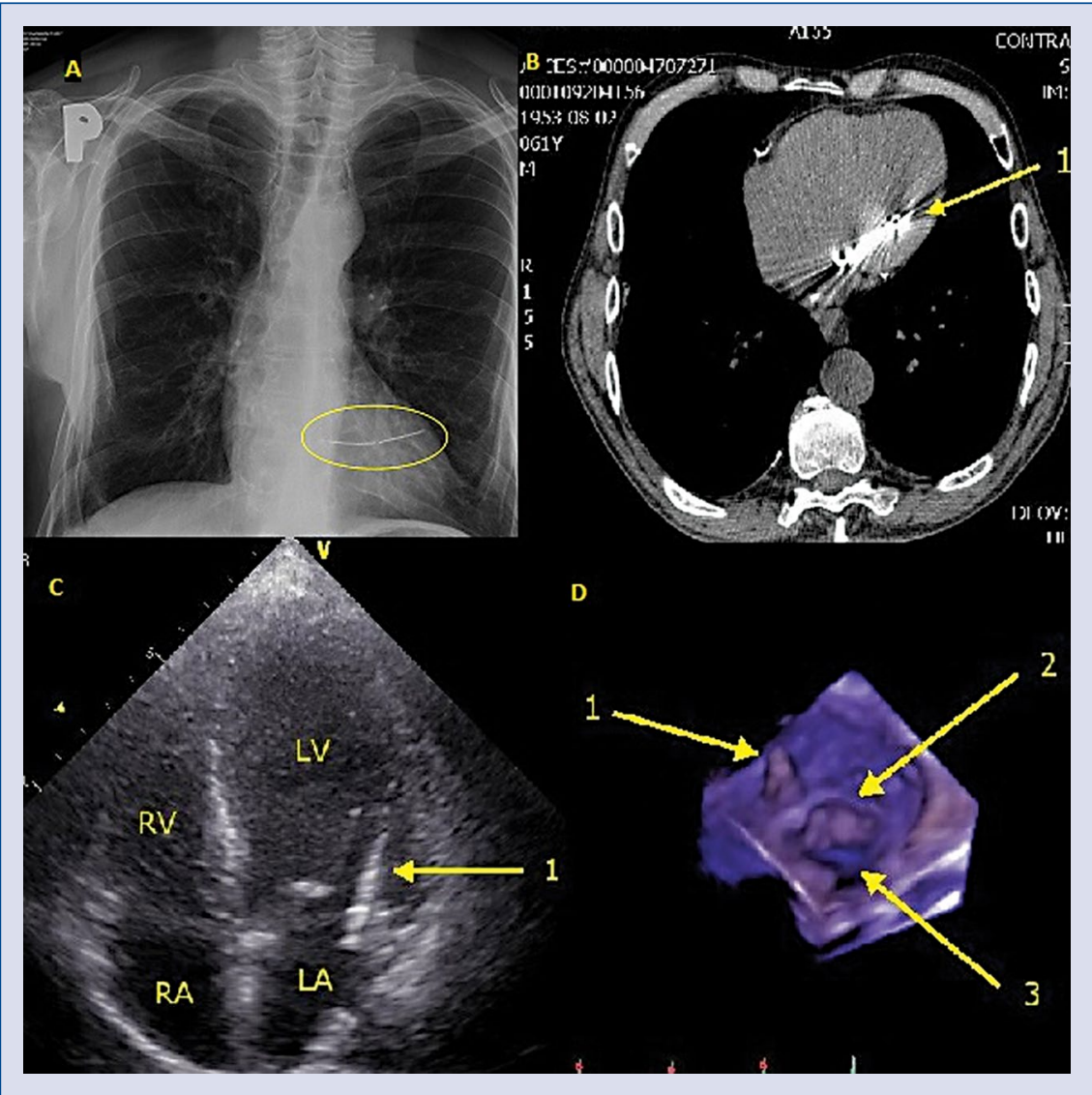

Figure 1. The foreign body (arrow 1) in antero-posterior chest X-ray (A), computed tomography scan (B), and echocardiography: two-dimensional (C, 4-chamber apical view), and 4-dimensional (D, arrow 2 - anterior mitral leaflet, arrow 3 - mitral orifice); LA — left atrium; LV — left ventricle; RA — right atrium; RV — right ventricle.

Address for correspondence: Dr. Marcin Sadowski, Faculty of Medicine and Health Sciences, Jan Kochanowski University, Al. IX Wieków Kielc 19A, 25-317 Kielce, Poland, e-mail: emsad@02.pl

Received: 10.07.2017 Accepted: 14.09.2017 
cup. He remembered that he felt extremely weak or even fainted then. The patient did not consent to any invasive diagnostic procedures, therefore only imaging studies were performed. Chest computed tomography scanning confirmed the presence of a metal foreign body of about $60 \mathrm{~mm}$ in length in the left ventricle (Fig. 1B). Additionally, echocardiography showed the presence of a linear foreign body lying along the lateral wall of the left ventricle and crossing the atrioventricular septum right behind the posterior mitral leaflet (Fig. 1C, D). Despite encouragement on several occasions the patient did not consent to removal of the foreign object from his heart. Whether the foreign body was responsible for the patient's condition remains unclear. Nevertheless, even surprising findings should not distract attending physicians from systematic patient evaluation.

Conflict of interest: None declared 